\title{
Size-Dependent Free Vibration of Silicon Nanobeams with Different Boundary Conditions and Beam Theories
}

\author{
B. UzUN* AND M.Ö. YAYLI \\ Bursa Uludag University, Engineering Faculty, Department of Civil Engineering, \\ Görükle Campus, 16059, Bursa, Turkey \\ Received: 31.03.2021 \& Accepted: 17.06.2021
}

Doi: 10.12693/APhysPolA.140.161

*e-mail: buzun@uludag.edu.tr

\begin{abstract}
This paper aims to investigate the size effect on the free vibration responses of nanobeams with various boundary conditions, especially guide supported boundary conditions. It is seen that the boundary conditions examined in the previously published articles are mostly clamped-clamped, simply supported at both ends and clamped-simply supported. The difference of this article is that it examines the size effect based on the modified couple stress theory on vibrations of nanobeams with guide supported boundary conditions as well. In addition, the influences of the cross-section and the rotary inertia effect change on the vibrational responses of the nanobeams are pursued as a case study. A finite element method procedure is utilized to calculate the free vibrational frequencies of nanobeams.
\end{abstract}

topics: guide supported nanobeam, modified couple stress theory, vibration, finite element method

\section{Introduction}

Nanotechnology, which is today's favorite technology, deals with the production of extremely small materials, understanding of their properties, usage areas, and possibilities. These extremely small materials, structures and structural elements are called nanomaterials and micromaterials. Researchers who study nanotechnology have presented various scientific papers [1-7] covering these materials' properties, and various responses in some conditions and areas of usability. The superior physical, chemical and mechanical properties of these materials have led to their utilization in a variety of applications in many disciplines, hence the realistic analysis of their mechanical responses is crucial. The atoms and molecules that compose the nanoand micromaterials eventuate in the small size effect in these structures. Therefore, non-classical continuum theories inclined to capture the size effects are more suitable for modeling and investigating the mechanical behaviors of extremely small micro- and nanostructures. These non-classical theories include new length scale parameters in addition to those in the classical theory to capture the small size effect. Many scientific studies based on various investigations of nano/microscaled elements conducted with non-classical elasticity theories such as the nonlocal elasticity theory [8-36], the strain gradient elasticity theory [37-43], the nonlocal couple stress theory [44], the surface elasticity theory $[45,46]$, the nonlocal strain gradient theory [47-58] etc. have been carried out by researchers in recent years.

One of these non-classical elasticity theories is the modified couple stress theory (MCST) which contains only one length scale parameter, the material length scale parameter, presented by Yang et al. [59]. Ghanbari and Babaei [60] have proposed the vibration analysis of a clamped-free microbeam supporting an attached mass in conjunction with MCST. The vibration behavior of a EulerBernoulli nanobeam, simply supported at both ends and resting on the one-parameter Winkler type elastic foundation, has been presented by Togun and Bağdatlı [61] based on MCST. Park and Gao [62], in turn, have presented the bending of a Bernoulli-Euler beam based on MCST. Kong et al. [63] analytically presented the dynamic problems of simply supported and cantilever Bernoulli-Euler microbeams in conjunction with MCST. Further, Civalek et al. [64] studied the free vibration characteristic of carbon nanotube-reinforced composite microscaled beams in the context of the modified couple stress theory based on various beam theories.

MCST has been used by researchers for analyses of various beam theories. Ersoy et al. [65] have analytically studied the free vibration responses of carbon nanotubes (CNTs) based on MCST and Rayleigh beam theories. Daneshmehr et al. [66] 
have presented thermal static bending, vibration and buckling responses of a microscale Reddy beam with simply supported boundary condition on the basis of MCST. Further, Ke et al. [67] have investigated the thermal effect on the stability and vibration behaviors of Timoshenko microbeams on the basis of MCST. Ansari et al. [68] have examined the vibration response of piezoelectric Euler-Bernoulli and Timoshenko microbeams in conjunction with MCST. Mazur et al. [69], in turn, have investigated the buckling and vibration behaviors of a microsized plate according to MCST and the KirchhoffLove plate theory. Akbaş [70] presented the forced vibration of a viscoelastic nanoscaled beam resting on the Winkler-Pasternak elastic foundation in the framework of MCST and the Timoshenko beam theory. In the study, the viscoelastic nanoscaled beam was modeled with simply supported boundary conditions and its forced vibration response was examined by using the finite element method. Uzun et al. [71] investigated the vibrational frequencies of single-walled carbon nanotube (SWCNT) based cantilever sensors in conjunction with the Rayleigh beam theory and MCST using the finite element method.

Also, MCST has been used in a nonlinear analysis of structures and structural elements. Asghari et al. [72] have presented an investigation of static bending and free vibration responses of a Timoshenko beam in conjunction with MCST. The nonlinear forced dynamics of a microbeam have been investigated numerically by Ghayesh et al. [73] based on MCST. Further, Wang et al. [74] have studied the nonlinear free vibration analysis of the microbeams in conjunction with the modified couple stress Euler-Bernoulli beam theory and the von Karman geometrically nonlinear theory. Togun and Bagdatli [75] have investigated the nonlinear vibrations of the tensioned nanobeams with clampedclamped and simply supported-simply supported boundary conditions via MCST.

Beams are among the primary structural elements of civil engineering and its structures. At the same time, it is a common situation in other engineering departments like automotive, aerospace, mechanical etc. to design various components as beam elements. Opportunities offered by nanotechnology, the favorite technology of recent years, have paved the way for the modeling of nanoscale beam structures. Nanomechanical resonators and sensors can be given as examples of nanoscale beam structures. It is known that classical elasticity theories do not realistically explicate the mechanical properties and behaviors of extremely small structures and structural elements.

This paper deals with the investigation of a size-dependent vibrational response of nanobeams with five different boundary conditions like clamped-clamped (C-C), simply supported at both ends (S-S) clamped--simply supported (C-S), simply supported-guide supported (S-G), and
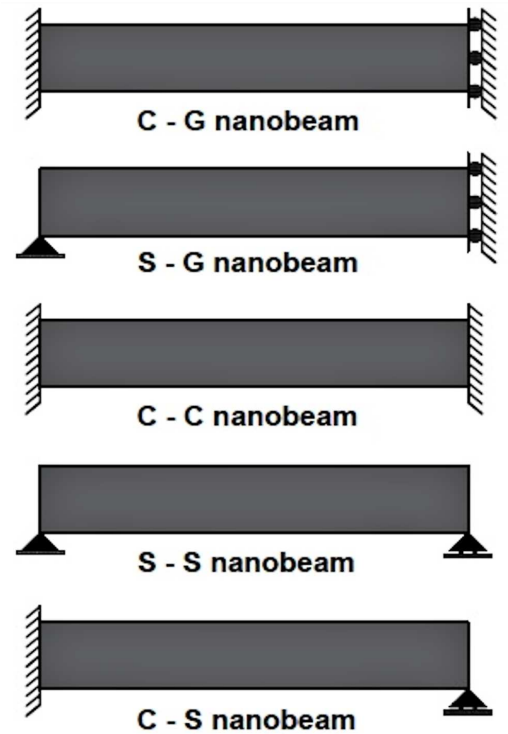

Fig. 1. Nanobeams with various boundary conditions.

clamped-guide supported (C-G). Size dependence of the nanobeam is handled based on MCST which has one additional length scale parameter. The finite element method is applied to solve the vibration problem of nanobeams. Effects of mode numbers, material length scale parameter and length of the beam (in other words, the lengthto-diameter/height ratio) on frequency values are investigated and compared for five kinds of boundary conditions (see Fig. 1). Also, the variations of frequencies of circular and rectangular simply supported nanobeams, including the rotary inertia effect, are investigated in this study.

\section{Euler-Bernoulli beam theory}

For the Euler-Bernoulli beam theory (classical beam theory), the displacement fields $u_{1}, u_{2}$ and $u_{3}$ are given as follows [16, 60, 62]:

$$
\begin{aligned}
& u_{1}(x, z, t)=-z \frac{\partial w(x, t)}{\partial x}, \\
& u_{2}(x, z, t)=0 \\
& u_{3}(x, z, t)=w(x, t) .
\end{aligned}
$$

where $x, y$ and $z$ direction indicate the length, width and height directions of the beam, respectively. In the above, $w(x, z, t)$ refers to transverse displacements of any point on the midline and $t$ denotes time. The strain tensor $\epsilon$ is stated as below

$$
\epsilon_{i j}=\frac{1}{2}\left(u_{i, j}+u_{j, i}\right)
$$

Substituting (1)-(3) into (4), the constituents of the strain tensor of the Euler-Bernoulli beam can be acquired as follows:

$$
\epsilon_{x x}=\frac{1}{2}\left(\frac{\partial u_{1}}{\partial x} \frac{\partial u_{1}}{\partial x}\right)=-z \frac{\partial^{2} w(x, t)}{\partial x^{2}},
$$




$$
\begin{aligned}
& \epsilon_{x y}=\epsilon_{y x}=\epsilon_{x z}=\epsilon_{z x}=0 \\
& \epsilon_{y y}=\epsilon_{y z}=\epsilon_{z y}=\epsilon_{z z}=0 .
\end{aligned}
$$

\section{Modified couple stress theory}

The modified couple stress theory (MCST), which can capture the size effect thanks to the material length scale parameter included in their formulas, was introduced by Yang et al. [59]. In MCST, strain energy density is defined as a function of both strain and curvature tensors. The strain energy $U$ of a linear elastic isotropic body occupying volume $V$ can be written as [59]:

$$
U=\frac{1}{2} \int \mathrm{d} V\left(\sigma_{i j} \epsilon_{i j}+m_{i j} \chi_{i j}\right)
$$

Here, $\sigma_{i j}$ denotes the classical stress tensor, while $m_{i j}$ and $\chi_{i j}$ indicate the symmetric couple stress tensor and the symmetric rotation gradient tensor, respectively. They are defined as [59]:

$$
\begin{aligned}
\sigma_{i j} & =\lambda \epsilon_{i i} \delta_{i j}+2 G \epsilon_{i j}, \\
m_{i j} & =2 G l^{2} \chi_{i j}, \\
\chi_{i j} & =\frac{1}{2}\left(\theta_{i, j}+\theta_{j, i}\right) .
\end{aligned}
$$

Here, $\lambda$ and $G$ represent the Lame coefficients, $\delta_{i j}$ is the Kronecker delta and $\theta$ is the rotation vector. In (9), $l$ specifies the material length scale parameter which provides the size effect to be captured. The Lame constants and the rotation vector are given as

$$
\begin{aligned}
\lambda & =\frac{E \nu}{(1+\nu)(1-2 \nu)}, \\
G & =\frac{1}{2} \frac{E}{(1+\nu)}, \\
\theta_{i} & =\frac{1}{2} e_{i j k} u_{k, j} .
\end{aligned}
$$

Here, $E$ and $v$ indicate Young's modulus and Poisson's ratio, respectively. The permutation symbol $e_{i j k}$ is defined as:

$$
e_{i j k}=\left\{\begin{array}{l}
+1 \text { when }(i j k)=\{(123),(231),(312)\} \\
-1 \text { when }(i j k)=\{(132),(213),(321)\}
\end{array}\right.
$$

Substituting (1)-(3) and (14) into (13), one obtains the components of the rotation vector, i.e.

$$
\begin{aligned}
& \theta_{1}=\frac{1}{2}\left(e_{123} \frac{\partial u_{3}}{\partial y}+e_{132} \frac{\partial u_{2}}{\partial z}\right)=0, \\
& \theta_{2}=\frac{1}{2}\left(e_{231} \frac{\partial u_{1}}{\partial z}+e_{213} \frac{\partial u_{3}}{\partial x}\right)= \\
& \frac{1}{2}\left((+1)\left(-\frac{\partial w}{\partial x}\right)+(-1)\left(-\frac{\partial w}{\partial x}\right)\right)=-\frac{\partial w}{\partial x}, \\
& \theta_{3}=\frac{1}{2}\left(e_{312} \frac{\partial u_{2}}{\partial x}+e_{321} \frac{\partial u_{1}}{\partial y}\right)=0 .
\end{aligned}
$$

Substituting (15) into (10), the following components of the symmetric rotation gradient tensor are achieved:

$$
\begin{aligned}
\chi_{11} & =\frac{1}{2}\left(\frac{\partial \theta_{1}}{\partial x}+\frac{\partial \theta_{1}}{\partial x}\right)=0 \\
\chi_{12} & =\frac{1}{2}\left(\frac{\partial \theta_{1}}{\partial y}+\frac{\partial \theta_{2}}{\partial x}\right)= \\
\frac{1}{2} & \left(0-\frac{\partial^{2} w}{\partial^{2} x}\right)=-\frac{1}{2} \frac{\partial^{2} w}{\partial^{2} x} \\
\chi_{13} & =\frac{1}{2}\left(\frac{\partial \theta_{1}}{\partial z}+\frac{\partial \theta_{3}}{\partial x}\right)=0 \\
\chi_{21} & =\chi_{12}, \\
\chi_{22} & =\frac{1}{2}\left(\frac{\partial \theta_{2}}{\partial y}+\frac{\partial \theta_{2}}{\partial y}\right)=0 \\
\chi_{23} & =\frac{1}{2}\left(\frac{\partial \theta_{2}}{\partial z}+\frac{\partial \theta_{3}}{\partial y}\right)=0 \\
\chi_{31} & =\frac{1}{2}\left(\frac{\partial \theta_{1}}{\partial z}+\frac{\partial \theta_{3}}{\partial x}\right)=0 \\
\chi_{32} & =\frac{1}{2}\left(\frac{\partial \theta_{2}}{\partial z}+\frac{\partial \theta_{3}}{\partial y}\right)=0 \\
& \frac{1}{2}\left(\frac{\partial \theta_{3}}{\partial z}+\frac{\partial \theta_{3}}{\partial z}\right)=0
\end{aligned}
$$

If $(5),(11),(12), \quad(15), \quad(16)$ are written into (8) and (9), one can obtain the classical stress tensors and symmetric couple stress tensors as follows:

$$
\begin{aligned}
& \sigma_{11}=-E z \frac{\partial^{2} w}{\partial x^{2}}, \\
& \sigma_{12}=\sigma_{21}=\sigma_{13}=\sigma_{31}=0, \\
& \sigma_{22}=\sigma_{33}=\sigma_{23}=\sigma_{32}=0, \\
& m_{12}=m_{21}=-G l^{2} \frac{\partial^{2} w}{\partial x^{2}}, \\
& m_{11}=m_{22}=m_{33}=0, \\
& m_{13}=m_{31}=m_{23}=m_{32}=0 .
\end{aligned}
$$

In the case of (17), it should be noted that the classical stress tensor is obtained by neglecting the Poisson effect. Substituting (5), (16), (17) and (19) into (7), the strain energy is provided as [63]:

$$
U=\frac{1}{2} \int_{0}^{L} \mathrm{~d} x\left(E I+G A l^{2}\right)\left(\frac{\partial^{2} w}{\partial x^{2}}\right)^{2} .
$$

In the strain energy formula, $A$ indicates the crosssection area of the beam and $I$ represents the moment of inertia. The kinetic energy of the nanobeam is defined as: 


$$
\begin{aligned}
K= & \frac{1}{2} \int_{V} \mathrm{~d} V \rho\left[\left(\frac{\partial u_{1}}{\partial t}\right)^{2}+\left(\frac{\partial u_{2}}{\partial t}\right)^{2}+\left(\frac{\partial u_{3}}{\partial t}\right)^{2}\right]= \\
& \frac{1}{2} \int_{0}^{L} \mathrm{~d} x\left[\rho A\left(\frac{\partial w}{\partial t}\right)^{2}+\rho I\left(\frac{\partial^{2} w}{\partial x \partial t}\right)^{2}\right] .
\end{aligned}
$$

Here, $\rho$ is the mass density of the beam. Hamilton's principle is used to derive the equation of motion of the nanobeam. It is expressed as [76]:

$$
\int_{t_{1}}^{t_{2}} \mathrm{~d} t(\delta K-\delta U+\delta W)=0 .
$$

In (23), $K, U$ and $W$ are the kinetic energy, strain energy and work done by external forces, respectively. In this vibration problem, there is no external force and consequently $W$ is equivalent to zero $(W=0)$. The first variation of the strain energy for nanobeam on the time interval $\left[t_{1}, t_{2}\right]$ can be given as

$$
\begin{aligned}
& \delta \int_{t_{1}}^{t_{2}} \mathrm{~d} t U= \\
& \int_{t_{1}}^{t_{2}} \mathrm{~d} t \int_{0}^{L} \mathrm{~d} x\left(E I+G A l^{2}\right) \frac{\partial^{2} w}{\partial x^{2}} \delta\left(\frac{\partial^{2} w}{\partial x^{2}}\right) .
\end{aligned}
$$

$$
\begin{aligned}
& -\int_{t_{1}}^{t_{2}} \mathrm{~d} t \int_{0}^{L} \mathrm{~d} x \rho A \frac{\partial^{2} w}{\partial t^{2}}(\delta w)+\left.\int_{0}^{L} \mathrm{~d} x \rho A \frac{\partial w}{\partial t}(\delta w)\right|_{t_{1}} ^{t_{2}}+\int_{t_{1}}^{t_{2}} \mathrm{~d} t \int_{0}^{L} \mathrm{~d} x \rho I \frac{\partial^{4} w}{\partial x^{2} \partial t^{2}}(\delta w) \\
& \quad+\left.\int_{0}^{L} \mathrm{~d} x \rho I \frac{\partial^{2} w}{\partial x \partial t} \delta\left(\frac{\partial w}{\partial x}\right)\right|_{t_{1}} ^{t_{2}}-\left.\int_{0}^{L} \mathrm{~d} x \rho I \frac{\partial^{3} w}{\partial x \partial t^{2}}(\delta w)\right|_{t_{1}} ^{t_{2}} .
\end{aligned}
$$

Using partial integration in (24), one gets

$$
\begin{aligned}
& \left.\int_{t_{1}}^{t_{2}} \mathrm{~d} t\left(E I+G A l^{2}\right) \frac{\partial^{2} w}{\partial x^{2}} \delta\left(\frac{\partial w}{\partial x}\right)\right|_{0} ^{L} \\
& -\left.\int_{t_{1}}^{t_{2}} \mathrm{~d} t\left(E I+G A l^{2}\right) \frac{\partial^{3} w}{\partial x^{3}}(\delta w)\right|_{0} ^{L} \\
& +\int_{t_{1}}^{t_{2}} \mathrm{~d} t \int_{0}^{L} \mathrm{~d} x\left(E I+G A l^{2}\right) \frac{\partial^{4} w}{\partial x^{4}}(\delta w)
\end{aligned}
$$

The first variation of the kinetic energy for the nanobeam on the time interval $\left[t_{1}, t_{2}\right]$ can be

$$
\begin{gathered}
\delta\left(\int_{t_{1}}^{t_{2}} \mathrm{~d} t K\right)=\int_{t_{1}}^{t_{2}} \mathrm{~d} t \int_{0}^{L} \mathrm{~d} x \rho A \frac{\partial w}{\partial t} \delta\left(\frac{\partial w}{\partial t}\right) \\
+\int_{t_{1}}^{t_{2}} \mathrm{~d} t \int_{0}^{L} \mathrm{~d} x \rho I \frac{\partial^{2} w}{\partial x \partial t} \delta\left(\frac{\partial^{2} w}{\partial x \partial t}\right)=0 .
\end{gathered}
$$

By using partial integration in (26), one obtains

If (25) and (27) are inserted into (23), one obtains

$$
\begin{aligned}
& \int_{t_{1}}^{t_{2}} \mathrm{~d} t \int_{0}^{L} \mathrm{~d} x\left[-\rho A \frac{\partial^{2} w}{\partial t^{2}}+\rho I \frac{\partial^{4} w}{\partial x^{2} \partial t^{2}}-\left(E I+G A l^{2}\right) \frac{\partial^{4} w}{\partial x^{4}}\right](\delta w)-\left.\int_{t_{1}}^{t_{2}} \mathrm{~d} t\left(E I+G A l^{2}\right) \frac{\partial^{2} w}{\partial x^{2}} \delta\left(\frac{\partial w}{\partial x}\right)\right|_{0} ^{L} \\
& \quad+\left.\int_{t_{1}}^{t_{2}} \mathrm{~d} t\left(E I+G A l^{2}\right) \frac{\partial^{3} w}{\partial x^{3}}(\delta w)\right|_{0} ^{L}+\left.\int_{0}^{L} \mathrm{~d} x \rho A \frac{\partial w}{\partial t}(\delta w)\right|_{t_{1}} ^{t_{2}}+\left.\int_{0}^{L} \mathrm{~d} x \rho I \frac{\partial^{2} w}{\partial x \partial t} \delta\left(\frac{\partial w}{\partial x}\right)\right|_{t_{1}} ^{t_{2}} \\
& \quad-\left.\int_{0}^{L} \mathrm{~d} x \rho \frac{\partial^{3} w}{\partial x \partial t^{2}}(\delta w)\right|_{t_{1}} ^{t_{2}}=0
\end{aligned}
$$

The equation of motion governing the nanobeam comes, due to the fundamental assumptions, from setting to zero the $\delta w$ variations within $w$. Therefore, the above calculation simplifies to $[65]$ : 


\section{Finite element method}

In this study, the finite element method procedure is used to perform the vibration analysis of the nanobeam based on MCST, including the rotary inertia effect. The beam element is modeled using two nodes and two degrees of freedom per node, as demonstrated in Fig. 2. The degrees of freedom of a beam element are $w_{1}$ (displacement of node 1 ), $\theta_{1}$ (rotation of node 1 ), $w_{2}$ (displacement of node 2 ), $\theta_{2}$ (rotation of node 2 ). In Fig. 3, a finite element illustration of the total beam is given. The parameters: $L, L_{\mathrm{e}}$ and $N$ represent the total length of the beam, length of a beam finite element and number of elements, respectively. The length of the beam finite element is found by dividing the total length of the beam by the number of finite elements.

Matrices that perform the finite element solution of the vibration problem are obtained by shape functions. The shape functions of the beam are defined as [77]:

$$
\phi=\left(\begin{array}{c}
\phi_{1} \\
\phi_{2} \\
\phi_{3} \\
\phi_{4}
\end{array}\right)=\left(\begin{array}{c}
1-\frac{3 x^{2}}{L_{\mathrm{e}}^{2}}+\frac{2 x^{3}}{L_{\mathrm{e}}^{3}} \\
x-\frac{2 x^{2}}{L_{\mathrm{e}}}+\frac{x^{3}}{L_{\mathrm{e}}^{2}} \\
\frac{3 x^{2}}{L_{\mathrm{e}}^{2}}-\frac{2 x^{3}}{L_{\mathrm{e}}^{3}} \\
-\frac{x^{2}}{L_{\mathrm{e}}}+\frac{x^{3}}{L_{\mathrm{e}}^{2}}
\end{array}\right)
$$

In order to get the weak form of the governing equation of the nanobeam based on MCST, the residue $R$ is expressed in the following way:

$$
R=E I \frac{\partial^{4} w}{\partial x^{4}}+G A l^{2} \frac{\partial^{4} w}{\partial x^{4}}+\rho A \frac{\partial^{2} w}{\partial t^{2}}-\rho I \frac{\partial^{4} w}{\partial x^{2} \partial t^{2}} .
$$

To determine the weighted residue, at first (31) is multiplied by a weighting function $(\phi)$ and next, it is integrated over the length $L$. Namely,

$$
\begin{aligned}
& \int_{0}^{L} \mathrm{~d} x \phi R=\int_{0}^{L} \mathrm{~d} x\left[\phi E I \frac{\partial^{4} w}{\partial x^{4}}+\phi G A l^{2} \frac{\partial^{4} w}{\partial x^{4}}\right. \\
& \left.+\phi \rho A \frac{\partial^{2} w}{\partial t^{2}}-\phi \rho I \frac{\partial^{4} w}{\partial x^{2} \partial t^{2}}\right]=0 .
\end{aligned}
$$

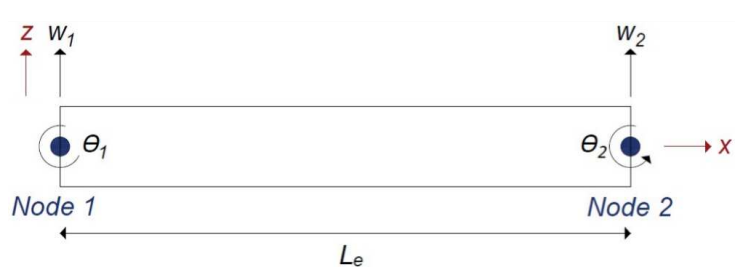

Fig. 2. A beam element and displacements.

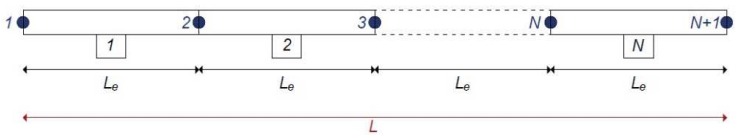

Fig. 3. A finite element illustration of the total beam.
With the use of partial integration in (32), one obtains

$$
\begin{aligned}
0= & \int_{0}^{L} \mathrm{~d} x\left[E I \ddot{\phi} \frac{\partial^{2} w}{\partial x^{2}}+G A l^{2} \dot{\phi} \frac{\partial^{2} w}{\partial x^{2}}+\rho A \phi \frac{\partial^{2} w}{\partial t^{2}}\right. \\
& \left.-\rho I \dot{\phi} \frac{\partial^{3} w}{\partial x \partial t^{2}}\right]+\left.\ldots E I \phi \frac{\partial^{3} w}{\partial x^{3}}\right|_{0} ^{L}-\left.E I \dot{\phi} \frac{\partial^{2} w}{\partial x^{2}}\right|_{0} ^{L} \\
& +\left.G A l^{2} \phi \frac{\partial^{3} w}{\partial x^{3}}\right|_{0} ^{L}-\left.G A l^{2} \dot{\phi} \frac{\partial^{2} w}{\partial x^{2}}\right|_{0} ^{L}-\left.\rho I \phi \frac{\partial^{3} w}{\partial x \partial t^{2}}\right|_{0} ^{L} .
\end{aligned}
$$

The general form of (33) can be written as

$$
\begin{gathered}
\int_{0}^{L} \mathrm{~d} x\left[E I \frac{\partial^{2} \phi}{\partial x^{2}} \frac{\partial^{2} \phi^{T}}{\partial x^{2}}+G A l^{2} \frac{\partial^{2} \phi}{\partial x^{2}} \frac{\partial^{2} \phi^{T}}{\partial x^{2}}+\rho A \phi \phi^{T}\right. \\
\left.-\rho I \frac{\partial \phi}{\partial x} \frac{\partial \phi^{T}}{\partial x}\right]=0
\end{gathered}
$$

From the substitution of the shape functions (30) into (34), the stiffness and mass matrices are obtained. It can therefore be understood that the Rayleigh beam has two stiffness matrices and two mass matrices. The stiffness and mass matrices belonging to the nanobeam are obtained as follows:

$$
\begin{aligned}
& K_{1}=E I \int_{0}^{L_{\mathrm{e}}} \mathrm{d} x\left(\begin{array}{c}
\ddot{\phi}_{1} \\
\ddot{\phi}_{2} \\
\ddot{\phi}_{3} \\
\ddot{\phi}_{4}
\end{array}\right)\left(\ddot{\phi}_{1}, \ddot{\phi}_{2}, \ddot{\phi}_{3}, \ddot{\phi}_{4}\right)= \\
& E I \int_{0}^{L_{\mathrm{e}}} \mathrm{d} x\left(\begin{array}{ccccc}
\ddot{\phi}_{1} \ddot{\phi}_{1} & \ddot{\phi}_{1} \ddot{\phi}_{2} & \ddot{\phi}_{1} \ddot{\phi}_{3} & \ddot{\phi}_{1} \ddot{\phi}_{4} \\
\ddot{\phi}_{2} \ddot{\phi}_{1} & \ddot{\phi}_{2} \ddot{\phi}_{2} & \ddot{\phi}_{2} \ddot{\phi}_{3} & \ddot{\phi}_{2} \ddot{\phi}_{4} \\
\ddot{\phi}_{3} \ddot{\phi}_{1} & \ddot{\phi}_{3} \ddot{\phi}_{2} & \ddot{\phi}_{3} \ddot{\phi}_{3} & \ddot{\phi}_{3} \ddot{\phi}_{4} \\
\ddot{\phi}_{4} \ddot{\phi}_{1} & \ddot{\phi}_{4} \ddot{\phi}_{2} & \ddot{\phi}_{4} \ddot{\phi}_{3} & \ddot{\phi}_{4} \ddot{\phi}_{4}
\end{array}\right)= \\
& \frac{E I}{L_{\mathrm{e}}^{3}}\left(\begin{array}{cccc}
12 & 6 L_{\mathrm{e}} & -12 & 6 L_{\mathrm{e}} \\
6 L_{\mathrm{e}} & 4 L_{\mathrm{e}}^{2} & -6 L_{\mathrm{e}} & 2 L_{\mathrm{e}}^{2} \\
-12 & -6 L_{\mathrm{e}} & 12 & -6 L_{\mathrm{e}} \\
6 L_{\mathrm{e}} & 2 L_{\mathrm{e}}^{2} & -6 L_{\mathrm{e}} & 4 L_{\mathrm{e}}^{2}
\end{array}\right) \\
& K_{2}=G A l^{2} \int_{0}^{L_{\mathrm{e}}} \mathrm{d} x\left(\begin{array}{c}
\ddot{\phi}_{1} \\
\ddot{\phi}_{2} \\
\ddot{\phi}_{3} \\
\ddot{\phi}_{4}
\end{array}\right)\left(\ddot{\phi}_{1}, \ddot{\phi}_{2}, \ddot{\phi}_{3}, \ddot{\phi}_{4}\right)= \\
& G A l^{2} \int_{0}^{L_{\mathrm{e}}} \mathrm{d} x\left(\begin{array}{ccccc}
\ddot{\phi}_{1} \ddot{\phi}_{1} & \ddot{\phi}_{1} \ddot{\phi}_{2} & \ddot{\phi}_{1} \ddot{\phi}_{3} & \ddot{\phi}_{1} \ddot{\phi}_{4} \\
\ddot{\phi}_{2} \ddot{\phi}_{1} & \ddot{\phi}_{2} \ddot{\phi}_{2} & \ddot{\phi}_{2} \ddot{\phi}_{3} & \ddot{\phi}_{2} \ddot{\phi}_{4} \\
\ddot{\phi}_{3} \ddot{\phi}_{1} & \ddot{\phi}_{3} \ddot{\phi}_{2} & \ddot{\phi}_{3} \ddot{\phi}_{3} & \ddot{\phi}_{3} \ddot{\phi}_{4} \\
\ddot{\phi}_{4} \ddot{\phi}_{1} & \ddot{\phi}_{4} \ddot{\phi}_{2} & \ddot{\phi}_{4} \ddot{\phi}_{3} & \ddot{\phi}_{4} \ddot{\phi}_{4}
\end{array}\right)= \\
& \frac{G A l^{2}}{L_{\mathrm{e}}^{3}}\left(\begin{array}{cccc}
12 & 6 L_{\mathrm{e}} & -12 & 6 L_{\mathrm{e}} \\
6 L_{\mathrm{e}} & 4 L_{\mathrm{e}}^{2} & -6 L_{\mathrm{e}} & 2 L_{\mathrm{e}}^{2} \\
-12 & -6 L_{\mathrm{e}} & 12 & -6 L_{\mathrm{e}} \\
6 L_{\mathrm{e}} & 2 L_{\mathrm{e}}^{2} & -6 L_{\mathrm{e}} & 4 L_{\mathrm{e}}^{2}
\end{array}\right)
\end{aligned}
$$




$$
\begin{aligned}
& M_{1}=\rho A \int_{0}^{L_{\mathrm{e}}} \mathrm{d} x\left(\begin{array}{c}
\phi_{1} \\
\phi_{2} \\
\phi_{3} \\
\phi_{4}
\end{array}\right)\left(\phi_{1}, \phi_{2}, \phi_{3}, \phi_{4}\right)= \\
& \rho A \int_{0}^{L_{\mathrm{e}}} \mathrm{d} x\left(\begin{array}{llll}
\phi_{1} \phi_{1} & \phi_{1} \phi_{2} & \phi_{1} \phi_{3} & \phi_{1} \phi_{4} \\
\phi_{2} \phi_{1} & \phi_{2} \phi_{2} & \phi_{2} \phi_{3} & \phi_{2} \phi_{4} \\
\phi_{3} \phi_{1} & \phi_{3} \phi_{2} & \phi_{3} \phi_{3} & \phi_{3} \phi_{4} \\
\phi_{4} \phi_{1} & \phi_{4} \phi_{2} & \phi_{4} \phi_{3} & \phi_{4} \phi_{4}
\end{array}\right)= \\
& \frac{\rho A}{420}\left(\begin{array}{cccc}
156 L_{\mathrm{e}} & 22 L_{\mathrm{e}} & 54 L_{\mathrm{e}} & -13 L_{\mathrm{e}}^{2} \\
22 L_{\mathrm{e}}^{2} & 4 L_{\mathrm{e}}^{3} & 13 L_{\mathrm{e}}^{2} & -3 L_{\mathrm{e}}^{3} \\
54 L_{\mathrm{e}} & 13 L_{\mathrm{e}}^{2} & 156 L_{\mathrm{e}} & -22 L_{\mathrm{e}}^{2} \\
-13 L_{\mathrm{e}}^{2} & -3 L_{\mathrm{e}}^{3} & -22 L_{\mathrm{e}}^{2} & 4 L_{\mathrm{e}}^{3}
\end{array}\right) \\
& M_{2}=\rho I \int_{0}^{L_{\mathrm{e}}} \mathrm{d} x\left(\begin{array}{c}
\dot{\phi}_{1} \\
\dot{\phi}_{2} \\
\dot{\phi}_{3} \\
\dot{\phi}_{4}
\end{array}\right)\left(\dot{\phi}_{1}, \dot{\phi}_{2}, \dot{\phi}_{3}, \dot{\phi}_{4}\right)= \\
& \rho I \int_{0}^{L_{\mathrm{e}}} \mathrm{d} x\left(\begin{array}{cccc}
\dot{\phi}_{1} \dot{\phi}_{1} & \dot{\phi}_{1} \dot{\phi}_{2} & \dot{\phi}_{1} \dot{\phi}_{3} & \dot{\phi}_{1} \dot{\phi}_{4} \\
\dot{\phi}_{2} \dot{\phi}_{1} & \dot{\phi}_{2} \dot{\phi}_{2} & \dot{\phi}_{2} \dot{\phi}_{3} & \dot{\phi}_{2} \dot{\phi}_{4} \\
\dot{\phi}_{3} \dot{\phi}_{1} & \dot{\phi}_{3} \dot{\phi}_{2} & \dot{\phi}_{3} \dot{\phi}_{3} & \dot{\phi}_{3} \dot{\phi}_{4} \\
\dot{\phi}_{4} \dot{\phi}_{1} & \dot{\phi}_{4} \dot{\phi}_{2} & \dot{\phi}_{4} \dot{\phi}_{3} & \dot{\phi}_{4} \dot{\phi}_{4}
\end{array}\right)= \\
& \frac{\rho I}{30 L_{\mathrm{e}}}\left(\begin{array}{cccc}
36 & 3 L_{\mathrm{e}} & -36 & 3 L_{\mathrm{e}} \\
3 L_{\mathrm{e}} & 4 L_{\mathrm{e}}^{2} & -3 L_{\mathrm{e}} & -L_{\mathrm{e}}^{2} \\
-36 & -3 L_{\mathrm{e}} & 36 & -3 L_{\mathrm{e}} \\
3 L_{\mathrm{e}} & -L_{\mathrm{e}}^{2} & -3 L_{\mathrm{e}} & 4 L_{\mathrm{e}}^{2}
\end{array}\right)
\end{aligned}
$$

The following defines the eigenvalue problem for a vibration analysis of a silicon nanobeam based on the Rayleigh beam theory. The eigenvalues $\left(\omega_{n}\right)$ may be calculated by setting the determinant to zero

$$
\left|K_{T}-\omega_{n}^{2} M_{T}\right|=0
$$

Here, $\omega$ represents the circular frequency, and the total stiffness $K_{T}$ and mass matrices $M_{T}$ are given as

$$
K_{T}=K_{1}+K_{2}
$$

and

$$
M_{T}=M_{1}+M_{2} .
$$

It is necessary to highlight a number of important points about the solution and theories performed. The previously mentioned total stiffness and mass matrices belong to the Rayleigh nanobeam based on MCST. In the total stiffness matrix, $K_{2}$ is a matrix coming from MCST which contains a material length scale parameter. And if $K_{2}$ is neglected, the finite element solution is simplified to the Rayleigh beam theory within the framework classical theory, which does not contain size dependence. In addition, $M_{2}$ is a matrix coming from the rotary inertia effect. When the rotary inertia effect is ignored by setting the value of $\rho I$ to zero, the solution is simplified to the Euler-Bernoulli beam theory based on MCST. If both $l$ and $\rho I$ are set to zero, the investigated problem is reduced to the vibration analysis of the Euler-Bernoulli beam according to the classical theory.

\section{Numerical results}

In the following section, a comparison study is provided to demonstrate the efficiency and validity of the method. A few numerical examples will be considered to emphasize the influence of the boundary conditions, cross-section, material length scale parameter $l$, length-to-diameter/height ratio $(L / D$ and $L / h)$ and rotary inertia. In this study, silicon $(\mathrm{Si})$ is modeled as a nanobeam, with different boundary conditions such as simply supported-simply supported, clamped-simply supported, clamped-clamped, clamped-guide supported and simply supported-guide supported. The parameters used in the calculations for silicon are as follows: Young's modulus $E=210 \mathrm{GPa}$, Poisson's ratio $\nu=0.24$ and mass density $\rho=$ $2370 \mathrm{~kg} / \mathrm{m}^{3}$ [78, 79]. The geometrical properties are selected as: diameter for a circular nanobeam $D=1 \mathrm{~nm}$, height for a rectangular nanobeam $h=$ $1 \mathrm{~nm}$ and length of the nanobeams $L=20-80 \mathrm{~nm}$.

For a simply supported-simply supported Rayleigh beam based on MCST, one can obtain the frequencies in the closed form by using

$$
\omega_{n}^{\text {Rayleigh }}=\frac{n^{2} \pi^{2}}{L^{2}} \sqrt{\frac{E I+G A l^{2}}{\rho A+\frac{n^{2} \pi^{2}}{L^{2}} \rho I}} .
$$

Applying (42) that comes from [65], the frequency values of a simply supported-simply supported Euler-Bernoulli beam based on MCST are found by setting $\rho I$ to zero. In Tables I and II, the results obtained from the frequency formula presented by Ersoy et al. [65] are compared with the results of the present study — found by using the finite element method for the different element numbers. For this comparison example, a circular nanobeam with simply supported boundary condition is considered and length of the nanobeam is selected to be $20 \mathrm{~nm}$. As can be seen in the comparison example, all results of the 28-element solution for both the Rayleigh and Euler-Bernoulli nanobeam match exactly.

The first five frequency values calculated from the vibration analyses of circular silicon nanobeams with S-S, C-G, S-G, C-C and C-S boundary conditions for various material length scale parameters ranging from 0 to 12 and length $L=20 \mathrm{~nm}$ are presented in Tables III-VII, respectively. It is apparently observed from these tables that the highest frequency values occurred for the C-C boundary condition, while the lowest values are seen in the S-G boundary condition. The obtained frequency values for different boundary conditions are listed from high to low as follows: $\mathrm{C}-\mathrm{C}>\mathrm{C}-\mathrm{S}>\mathrm{S}-\mathrm{S}>$ $\mathrm{C}-\mathrm{G}>\mathrm{S}-\mathrm{G}$. In addition, this order will be observed in the figures that will be presented later in this article. 
TABLE I

Comparison of the natural frequency $[\mathrm{GHz}]$ of a circular Euler-Bernoulli nanobeam for various length scale parameters.

\begin{tabular}{c|c|c|c|c|c|c|c|c|c|c}
\hline \hline \multirow{2}{*}{$l$} & \multirow{2}{*}{ Ersoy et al. [65] } & \multicolumn{8}{c}{ Element number $N$} \\
\cline { 3 - 10 } & & 8 & 9 & 10 & 11 & 12 & 13 & 14 & 15 & 28 \\
\hline 0 & 9.2413 & 9.2415 & 9.2414 & 9.2414 & 9.2414 & 9.2414 & 9.2414 & 9.2414 & 9.2414 & 9.2413 \\
0.1 & 9.5348 & 9.5350 & 9.5349 & 9.5349 & 9.5348 & 9.5348 & 9.5348 & 9.5348 & 9.5348 & 9.5348 \\
0.2 & 10.3654 & 10.3656 & 10.3655 & 10.3655 & 10.3655 & 10.3655 & 10.3654 & 10.3654 & 10.3654 & 10.3654
\end{tabular}

TABLE II

Comparison of the natural frequency $[\mathrm{GHz}]$ of a circular Rayleigh nanobeam for various length scale parameters.

\begin{tabular}{c|c|c|c|c|c|c|c|c|c|c}
\hline \hline \multirow{2}{*}{$l$} & \multirow{2}{*}{ Ersoy et al. [65] } & \multicolumn{8}{|c}{ Element number $N$} \\
\cline { 3 - 10 } & & 8 & 9 & 10 & 11 & 12 & 13 & 14 & 15 & 28 \\
\hline 0 & 9.2342 & 9.2344 & 9.2343 & 9.2343 & 9.2343 & 9.2343 & 9.2343 & 9.2342 & 9.2342 & 9.2342 \\
0.1 & 9.5275 & 9.5277 & 9.5276 & 9.5275 & 9.5275 & 9.5275 & 9.5275 & 9.5275 & 9.5275 & 9.5275 \\
0.2 & 10.3574 & 10.3576 & 10.3575 & 10.3575 & 10.3575 & 10.3575 & 10.3575 & 10.3575 & 10.3574 & 10.3574
\end{tabular}

TABLE III

First five frequencies $[\mathrm{GHz}]$ of circular silicon nanobeams with S-S boundary condition for various material length scale parameters $(L=20 \mathrm{~nm})$.

\begin{tabular}{c|c|c|c|c|c|c|c}
\hline \hline \multirow{2}{*}{$\omega_{n}[\mathrm{GHz}]$} & \multicolumn{7}{|c}{$l[\mathrm{~nm}]$} \\
\cline { 2 - 7 } & 0 & 2 & 4 & 6 & 8 & 10 & 12 \\
\hline$\omega_{1}$ & 9.2413 & 47.8470 & 94.3459 & 141.1412 & 188.0117 & 234.9123 & 281.8282 \\
$\omega_{2}$ & 36.9654 & 191.3881 & 377.3836 & 564.5647 & 752.0466 & 939.6494 & 1127.3126 \\
$\omega_{3}$ & 83.1721 & 430.6233 & 849.1131 & 1270.2706 & 1692.1050 & 2114.2112 & 2536.4535 \\
$\omega_{4}$ & 147.8616 & 765.5527 & 1509.5346 & 2258.2591 & 3008.1870 & 3758.5981 & 4509.2512 \\
$\omega_{5}$ & 231.0338 & 1196.1764 & 2358.6483 & 3528.5307 & 4700.2934 & 5872.8111 & 7045.7068
\end{tabular}

TABLE IV

First five frequencies $[\mathrm{GHz}]$ of circular silicon nanobeams with C-G boundary condition for various material length scale parameters $(L=20 \mathrm{~nm})$.

\begin{tabular}{c|c|c|c|c|c|c|c}
\hline \hline \multirow{2}{*}{$\omega_{n}[\mathrm{GHz}]$} & \multicolumn{7}{|c}{$l[\mathrm{~nm}]$} \\
\cline { 2 - 7 } & 0 & 2 & 4 & 6 & 8 & 10 & 12 \\
\hline$\omega_{1}$ & 5.2373 & 27.1160 & 53.4679 & 79.9878 & 106.5503 & 133.1300 & 159.7182 \\
$\omega_{2}$ & 28.3018 & 146.5324 & 288.9361 & 432.2475 & 575.7892 & 719.4234 & 863.1040 \\
$\omega_{3}$ & 69.8877 & 361.8432 & 713.4909 & 1067.3801 & 1421.8382 & 1776.5247 & 2131.3255 \\
$\omega_{4}$ & 129.9565 & 672.8490 & 1326.7393 & 1984.7979 & 2643.9143 & 3303.4553 & 3963.2089 \\
$\omega_{5}$ & 208.5080 & 1079.5491 & 2128.6799 & 3184.4987 & 4242.0144 & 5300.2116 & 6358.7499
\end{tabular}

TABLE V

First five frequencies $[\mathrm{GHz}]$ of circular silicon nanobeams with S-G boundary condition for various material length scale parameters $(L=20 \mathrm{~nm})$.

\begin{tabular}{c|c|c|c|c|c|c|c}
\hline \hline \multirow{2}{*}{$\omega_{n}[\mathrm{GHz}]$} & \multicolumn{7}{|c}{$l[\mathrm{~nm}]$} \\
\cline { 2 - 7 } & 0 & 2 & 4 & 6 & 8 & 10 & 12 \\
\hline$\omega_{1}$ & 2.3103 & 11.9618 & 23.5865 & 35.2853 & 47.0030 & 58.7281 & 70.4570 \\
$\omega_{2}$ & 20.7930 & 107.6558 & 212.2783 & 317.5676 & 423.0262 & 528.5528 & 634.1133 \\
$\omega_{3}$ & 57.7584 & 299.0440 & 589.6618 & 882.1323 & 1175.0729 & 1468.2022 & 1761.4260 \\
$\omega_{4}$ & 113.2065 & 586.1262 & 1155.7373 & 1728.9795 & 2303.1430 & 2877.6765 & 3452.3952 \\
$\omega_{5}$ & 187.1374 & 968.9027 & 1910.5049 & 2858.1094 & 3807.2371 & 4756.9763 & 5707.0217
\end{tabular}


TABLE VI

First five frequencies $[\mathrm{GHz}]$ of circular silicon nanobeams with C-C boundary condition for various material length scale parameters $(L=20 \mathrm{~nm})$.

\begin{tabular}{c|c|c|c|c|c|c|c}
\hline \hline \multirow{2}{*}{$\omega_{n}[\mathrm{GHz}]$} & \multicolumn{7}{|c}{$l[\mathrm{~nm}]$} \\
\cline { 2 - 8 } & 0 & 2 & 4 & 6 & 8 & 10 & 12 \\
\hline$\omega_{1}$ & 20.9491 & 108.4639 & 213.8716 & 319.9512 & 426.2013 & 532.5199 & 638.8728 \\
$\omega_{2}$ & 57.7470 & 298.9848 & 589.5452 & 881.9578 & 1174.8404 & 1467.9117 & 1761.0775 \\
$\omega_{3}$ & 113.2072 & 586.1298 & 1155.7444 & 1728.9900 & 2303.1571 & 2877.6941 & 3452.4163 \\
$\omega_{4}$ & 187.1373 & 968.9025 & 1910.5045 & 2858.1088 & 3807.2363 & 4756.9753 & 5707.0205 \\
$\omega_{5}$ & 279.5510 & 1447.3737 & 2853.9651 & 4269.5230 & 5687.3561 & 7106.1028 & 8525.3070
\end{tabular}

TABLE VII

First five frequencies $[\mathrm{GHz}]$ of circular silicon nanobeams with C-S boundary condition for various material length scale parameters $(L=20 \mathrm{~nm})$.

\begin{tabular}{c|c|c|c|c|c|c|c}
\hline \hline \multirow{2}{*}{$\omega_{n}[\mathrm{GHz}]$} & \multicolumn{7}{|c}{$l[\mathrm{~nm}]$} \\
\cline { 2 - 8 } & 0 & 2 & 4 & 6 & 8 & 10 & 12 \\
\hline$\omega_{1}$ & 14.4368 & 74.7462 & 147.3863 & 220.4894 & 293.7101 & 366.9779 & 440.2694 \\
$\omega_{2}$ & 46.7843 & 242.2256 & 477.6260 & 714.5270 & 951.8088 & 1189.2435 & 1426.7548 \\
$\omega_{3}$ & 97.6118 & 505.3843 & 996.5286 & 1490.8037 & 1985.8733 & 2481.2618 & 2976.8101 \\
$\omega_{4}$ & 166.9219 & 864.2373 & 1704.1231 & 2549.3629 & 3395.9613 & 4243.1051 & 5090.5221 \\
$\omega_{5}$ & 254.7148 & 1318.7846 & 2600.4100 & 3890.2054 & 5182.0739 & 6474.7748 & 7767.8925
\end{tabular}

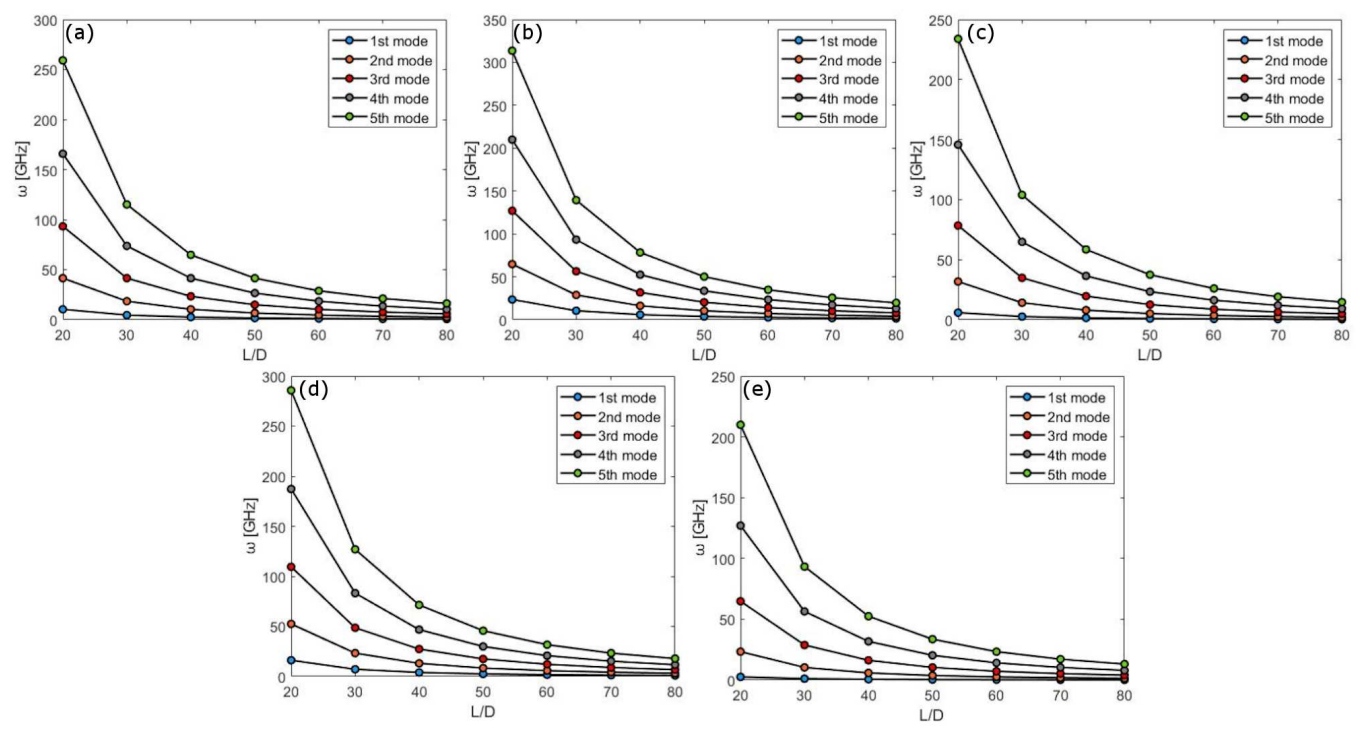

Fig. 4. Effect of the aspect ratio $L / D$ on variation of frequencies of a circular silicon nanobeam for different mode numbers $(l=0.2 \mathrm{~nm})$ : (a) S-S, (b) C-C, (c) C-G, (d) C-S, (e) S-G.

In Fig. 4a-e, for S-S, C-C, C-G, C-S and S-G circular silicon nanobeams, the variation of frequencies versus the changing aspect ratios $\mathrm{L} / \mathrm{D}$ are depicted for the first five modes. For these figures, the length scale parameter is taken as $l=0.2 \mathrm{~nm}$. The aim of these figures is to demonstrate the effect of the aspect ratio on the frequencies of a nanobeam for different $L / D$ values $(20,30,40,50,60,70,80)$. As it can be seen from Fig. $4 \mathrm{a}-\mathrm{e}$, by increasing the aspect ratio, the natural frequencies decrease dramatically for all of boundary conditions and all modes.
The frequencies of circular silicon nanobeams are plotted against the mode number for various material length scale parameters in Fig. 5a-e. In this example, material length scale parameters are chosen in the range of 0 and $12 \mathrm{~nm}$ to demonstrate the small-scale effect on the vibration analysis of the silicon nanobeam. It should be emphasized that when the material length scale parameter is set to zero $(l=0)$, the results of the local (classical) elasticity theory are obtained. It is revealed from Fig. $5 \mathrm{a}-\mathrm{e}$ that the material length scale parameter 
First five frequencies $[\mathrm{GHz}]$ of a circular Rayleigh nanobeam for various beam lengths $(l=0.2 \mathrm{~nm})$.

\begin{tabular}{c|c|c|c|c|c|c|c}
\hline \hline \multirow{2}{*}{$\omega_{n}[\mathrm{GHz}]$} & \multicolumn{7}{|c}{$L[\mathrm{~nm}]$} \\
\cline { 2 - 8 } & 20 & 30 & 40 & 50 & 60 & 70 & 80 \\
\hline$\omega_{1}$ & 10.3574 & 4.6053 & 2.5909 & 1.6583 & 1.1516 & 0.8461 & 0.6478 \\
$\omega_{2}$ & 41.3344 & 18.4022 & 10.3574 & 6.6306 & 4.6053 & 3.3838 & 2.5909 \\
$\omega_{3}$ & 92.6480 & 41.3344 & 23.2818 & 14.9097 & 10.3574 & 7.6111 & 5.8280 \\
$\omega_{4}$ & 163.8378 & 73.3088 & 41.3344 & 26.4833 & 18.4022 & 13.5249 & 10.3574 \\
$\omega_{5}$ & 254.2803 & 114.1971 & 64.4739 & 41.3344 & 28.7314 & 21.1207 & 16.1765
\end{tabular}

TABLE IX

First five frequencies $[\mathrm{GHz}]$ of a circular Euler-Bernoulli nanobeam for various beam lengths $(l=0.2 \mathrm{~nm})$.

\begin{tabular}{c|c|c|c|c|c|c|c}
\hline \hline \multirow{2}{*}{$\omega_{n}[\mathrm{GHz}]$} & \multicolumn{7}{|c}{$L[\mathrm{~nm}]$} \\
\cline { 2 - 8 } & 20 & 30 & 40 & 50 & 60 & 70 & 80 \\
\hline$\omega_{1}$ & 10.3654 & 4.6069 & 2.5914 & 1.6585 & 1.1517 & 0.8462 & 0.6478 \\
$\omega_{2}$ & 41.4617 & 18.4274 & 10.3654 & 6.6339 & 4.6069 & 3.3846 & 2.5914 \\
$\omega_{3}$ & 93.2888 & 41.4617 & 23.3222 & 14.9262 & 10.3654 & 7.6154 & 5.8305 \\
$\omega_{4}$ & 165.8467 & 73.7097 & 41.4617 & 26.5355 & 18.4274 & 13.5385 & 10.3654 \\
$\omega_{5}$ & 259.1356 & 115.1714 & 64.7839 & 41.4617 & 28.7928 & 21.1539 & 16.1960
\end{tabular}
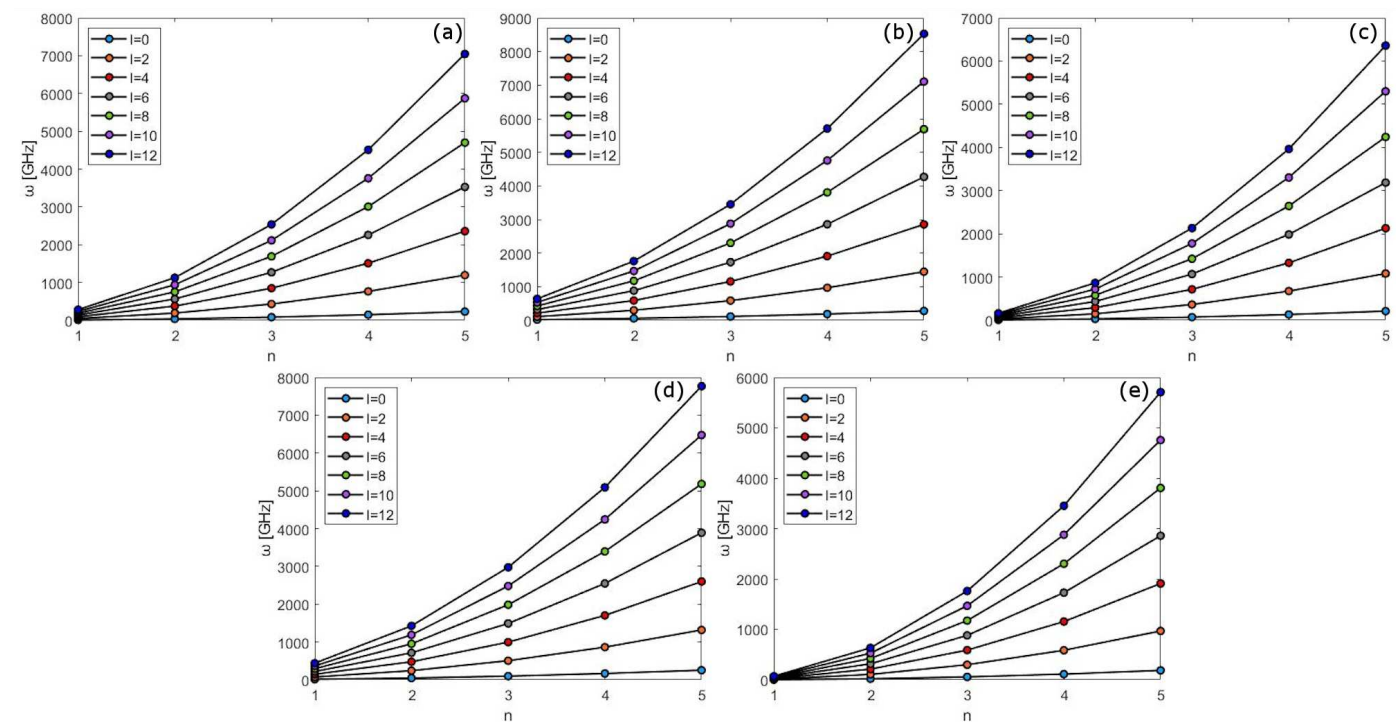

Fig. 5. Effect of the length scale parameter $l$ on variation of frequencies of a circular silicon nanobeam for different mode numbers $(L=20 \mathrm{~nm})$ : (a) S-S, (b) C-C, (c) C-G, (d) C-S, (e) S-G.

plays an important role in the frequency values of silicon nanobeams. Based on the results in these figures, the increasing value of the material length scale parameter causes an increase in the magnitude of frequencies.

To investigate the cross-section and rotary inertia effects, a silicon nanobeam simply supported at both ends is considered. Two types of a crosssection, circular and rectangular, with the same cross-sectional areas and height (diameter) are considered in this study to examine their frequency values and demonstrate differences between them. Frequency values obtained via finite element solutions of silicon nanobeams with circular and rectangular cross-sections (see Figs. 6 and 7) are compared with each other.

The first five frequency values calculated from the vibration analysis of a simply supported circular silicon nanobeam for various lengths $L$ ranging from 20 to 80 and the material length scale parameter $l=0.2$ are presented in Tables VIII and IX. In Tables VIII and IX, the first five frequencies of rectangular Rayleigh and Euler-Bernoulli silicon nanobeams for various length values are calculated, respectively. When the frequency values in Tables VIII-XI are compared, it can be seen that 
TABLE X

First five frequencies $[\mathrm{GHz}]$ of a rectangular Rayleigh nanobeam for various beam lengths $(l=0.2 \mathrm{~nm})$.

\begin{tabular}{c|c|c|c|c|c|c|c}
\hline \hline \multirow{2}{*}{$\omega_{n}[\mathrm{GHz}]$} & \multicolumn{7}{|c}{$L[\mathrm{~nm}]$} \\
\cline { 2 - 8 } & 20 & 30 & 40 & 50 & 60 & 70 & 80 \\
\hline$\omega_{1}$ & 11.6461 & 5.1790 & 2.9138 & 1.8650 & 1.2952 & 0.9516 & 0.7286 \\
$\omega_{2}$ & 46.4415 & 20.6876 & 11.6461 & 7.4562 & 5.1790 & 3.8054 & 2.9138 \\
$\omega_{3}$ & 103.9646 & 46.4415 & 26.1701 & 16.7628 & 11.6461 & 8.5586 & 6.5538 \\
$\omega_{4}$ & 183.5338 & 82.3020 & 46.4415 & 29.7663 & 20.6876 & 15.2064 & 11.6461 \\
$\omega_{5}$ & 284.2367 & 128.0788 & 72.3989 & 46.4415 & 32.2913 & 23.7421 & 18.1865
\end{tabular}

TABLE XI

First five frequencies $[\mathrm{GHz}]$ of a rectangular Euler-Bernoulli nanobeam for various beam lengths $(l=0.2 \mathrm{~nm})$

\begin{tabular}{c|c|c|c|c|c|c|c}
\hline \hline \multirow{2}{*}{$\omega_{n}[\mathrm{GHz}]$} & \multicolumn{7}{|c}{$L[\mathrm{~nm}]$} \\
\cline { 2 - 7 } & 20 & 30 & 40 & 50 & 60 & 70 & 80 \\
\hline$\omega_{1}$ & 11.6580 & 5.1813 & 2.9145 & 1.8653 & 1.2953 & 0.9517 & 0.7286 \\
$\omega_{2}$ & 46.6321 & 20.7254 & 11.6580 & 7.4611 & 5.1813 & 3.8067 & 2.9145 \\
$\omega_{3}$ & 104.9222 & 46.6321 & 26.2305 & 16.7875 & 11.6580 & 8.5651 & 6.5576 \\
$\omega_{4}$ & 186.5283 & 82.9015 & 46.6321 & 29.8445 & 20.7254 & 15.2268 & 11.6580 \\
$\omega_{5}$ & 291.4506 & 129.5336 & 72.8626 & 46.6321 & 32.3834 & 23.7919 & 18.2157
\end{tabular}

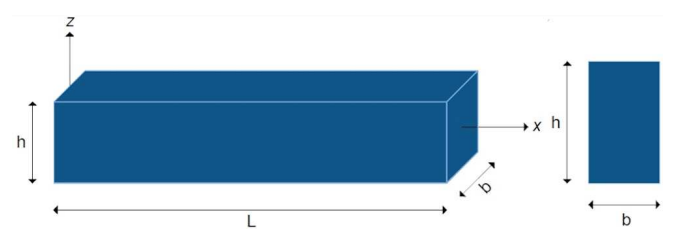

Fig. 6. A nanobeam with a rectangular crosssection.

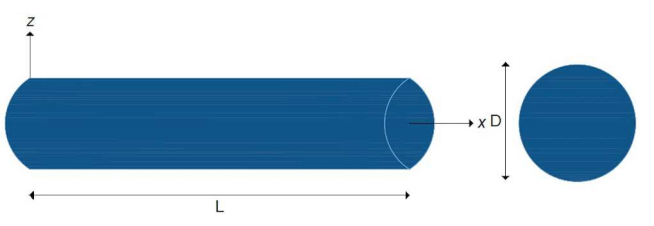

Fig. 7. A nanobeam with a circular cross-section.

the frequencies of the Rayleigh nanobeam are lower than the frequencies of the Euler nanobeam. However, when the results obtained at the first mode for the $80 \mathrm{~nm}$ beam length are examined, it is seen that the frequencies of the Euler-Bernoulli and Rayleigh beam theories are equalized. It can also be clearly seen from the tables that the frequencies of the nanobeam with a rectangular cross-section are higher than the frequencies of the nanobeam with a circular cross-section.

Variations of frequency values of a silicon nanobeam according to the Euler-Bernoulli and the Rayleigh beam theory versus the aspect ratio for the first five modes are shown in Figs. 8 and 9, respectively. Figures $8 \mathrm{a}$ and $9 \mathrm{a}$ show the results of a silicon nanobeam with a circular cross-section, while Figs $8 \mathrm{~b}$ and $9 \mathrm{~b}$ depict the results of a silicon nanobeam with a rectangular cross-section.
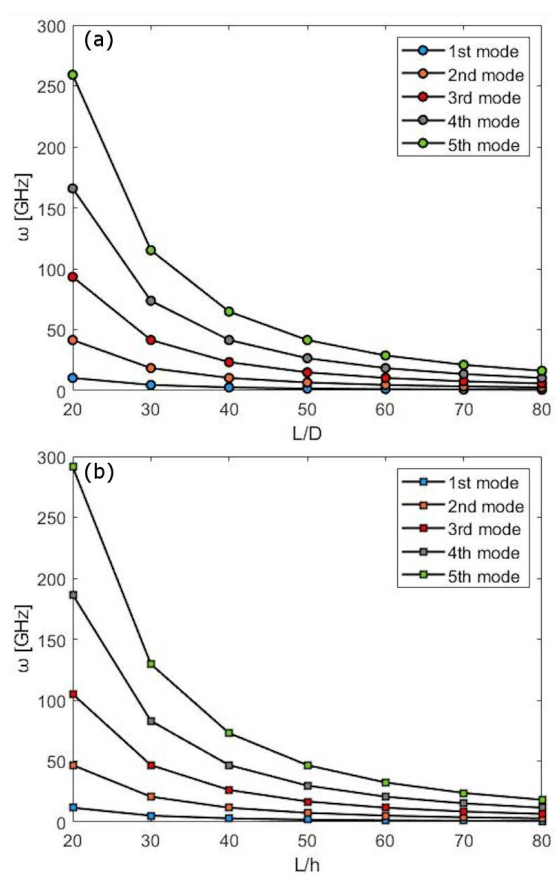

Fig. 8. Effect of the aspect ratio $L / D$ and $L / h$ on variation of frequencies of an Euler-Bernoulli nanobeam for different mode numbers $(l=0.2 \mathrm{~nm})$ :

(a) circular, (b) rectangular.

It can be clearly seen from these figures that the frequencies of the rectangular silicon nanobeam are higher than the frequencies of the circular silicon nanobeam. Even if the cross-sectional areas and heights of the nanobeams are the same, the frequencies of the nanobeam with a rectangular cross-section are higher as the moment of inertia is greater. 

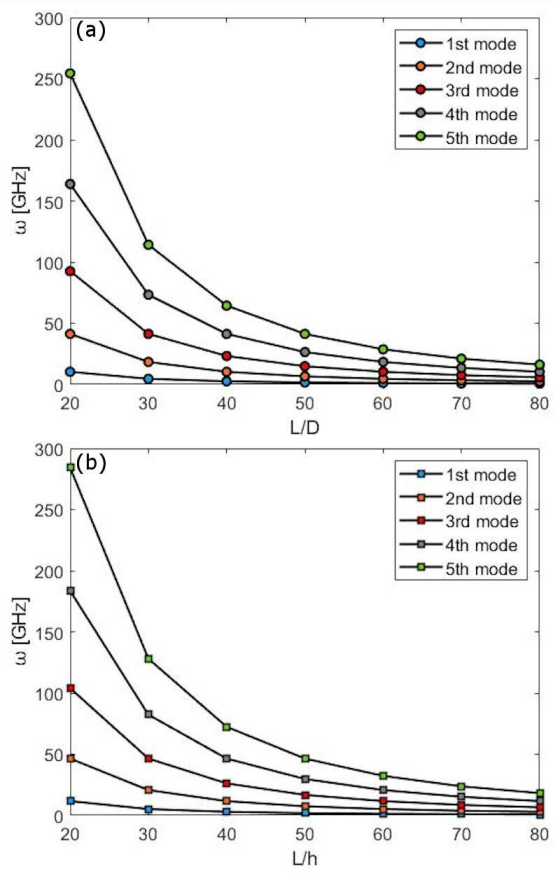

Fig. 9. Effect of the aspect ratio $L / D$ and $L / h$ on variation of frequencies of a Rayleigh nanobeam for different mode numbers $(l=0.2 \mathrm{~nm})$ : (a) circular, (b) rectangular.

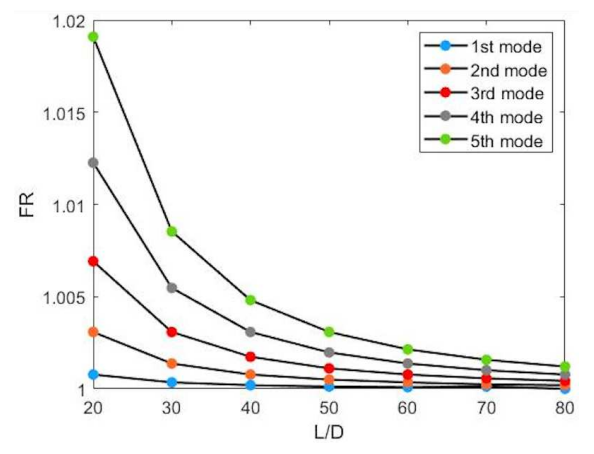

Fig. 10. Variation of frequency ratios of a circular nanobeam with the aspect ratio $L / D$ for different mode numbers $(l=0.2 \mathrm{~nm})$.

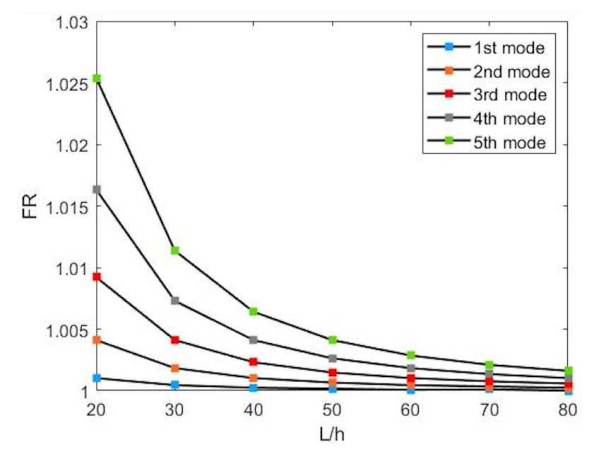

Fig. 11. Variation of frequency ratios of a rectangular nanobeam with the aspect ratio $L / h$ for different mode numbers $(l=0.2 \mathrm{~nm})$.
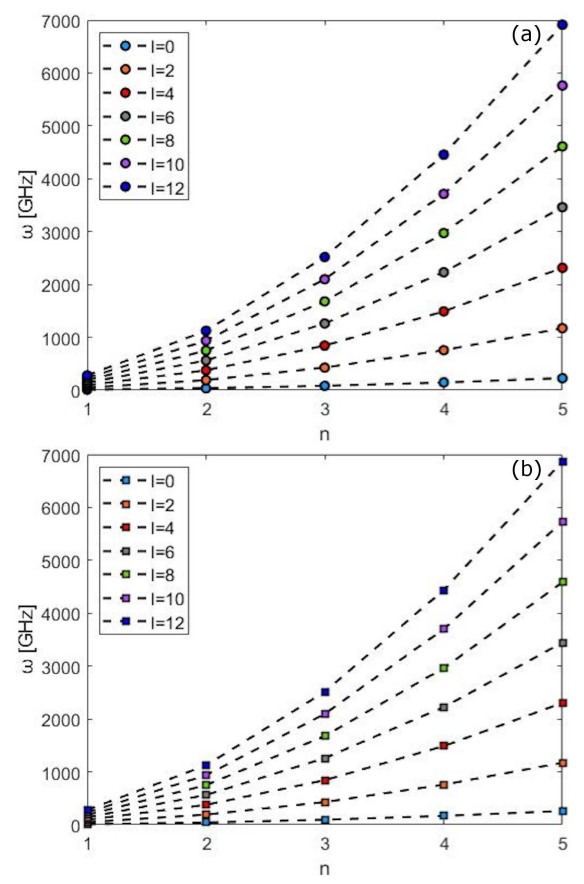

Fig. 12. Effect of the length scale parameter $l$ on variation of frequencies of a Rayleigh nanobeam for different mode numbers $(L=20 \mathrm{~nm})$ : (a) circular, (b) rectangular.

Figures 10 and 11 are plotted to show the influence of the aspect ratio on the frequency ratios $(F R)$ for the first five modes. The frequency ratio is defined as:

$$
F R=\frac{\omega_{\mathrm{E}}}{\omega_{\mathrm{R}}}
$$

where $\omega_{\mathrm{E}}$ and $\omega_{\mathrm{R}}$ represent the frequency values of the Euler-Bernoulli and the Rayleigh nanobeam, respectively.

One can observe from these figures that the frequency ratios of rectangular silicon nanobeams are higher than the frequency ratios of circular silicon nanobeams. In Figs. 10 and 11, it can be concluded that the effects of the aspect ratio increase with increasing mode number.

Variations of frequency values of the silicon nanobeam according to the Rayleigh and EulerBernoulli beam theories versus the mode number for various material length scale parameter values are shown in Figs. 12 and 13, respectively. It is understood that the influence of the material length scale parameter is more prominent for the silicon nanobeam with a circular cross-section. Figures 14 and 15 are plotted to better illustrate this effect. It is understood from Fig. 11 that the frequency values of the silicon nanobeam with a rectangular cross-section are higher than the frequency values of the silicon nanobeam with a circular cross-section while the material length scale parameter is small. As the material length scale parameter value increases, the difference between the frequencies of these nanobeams is closed. After a certain material length scale parameter value, the frequency 

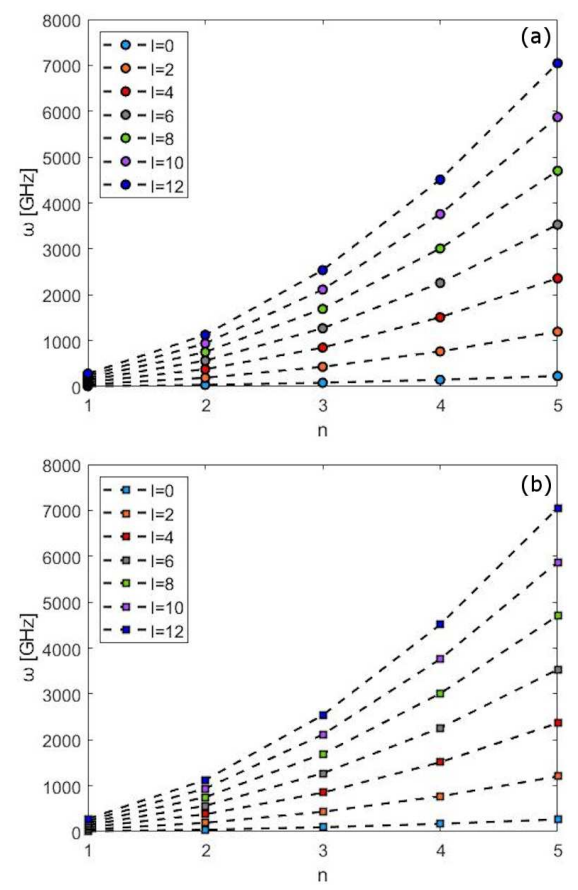

Fig. 13. Effect of the length scale parameter $l$ on variation of frequencies of an Euler-Bernoulli nanobeam for different mode numbers $(L=20 \mathrm{~nm})$ : (a) circular, (b) rectangular.

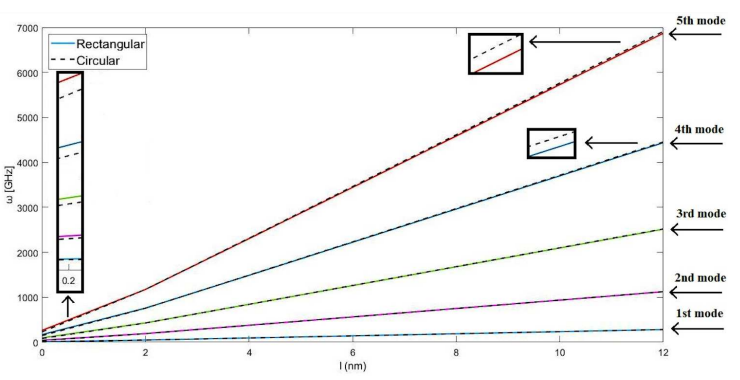

Fig. 14. Effect of the material length scale parameter $l$ on variation of frequencies of a Rayleigh nanobeam for different mode numbers $(L=20 \mathrm{~nm})$.

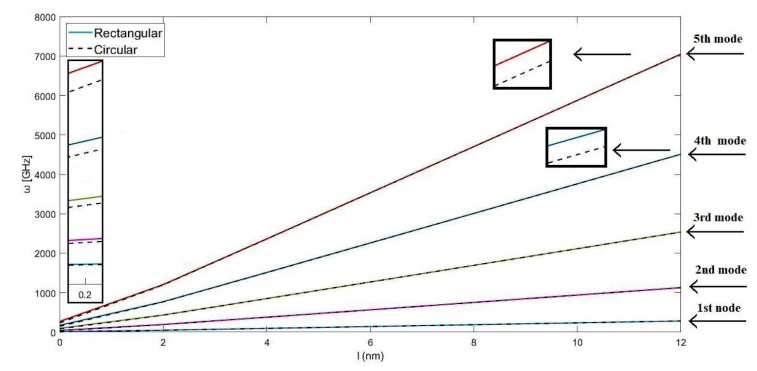

Fig. 15. Effect of the material length scale parameter $l$ on variation of frequencies of an EulerBernoulli nanobeam for different mode numbers $(L=20 \mathrm{~nm})$. values of the nanobeam with a circular cross-section become higher than the frequency values of the nanobeam with a rectangular cross-section. Among the material length scale parameter values examined $(l=0 \sim 12 \mathrm{~nm}$ ), this state is realized according to the Rayleigh beam theory, while in the Euler-Bernoulli beam theory, this situation does not occur. The frequency values of the nanobeam with a rectangular cross-section are always higher according to the Euler-Bernoulli beam theory.

\section{Conclusions}

In this work, the higher order elasticity theory, namely a modified couple stress theory, is used to investigate the vibration response of silicon nanobeams with various boundary conditions. Clamped-clamped, clamped-simply supported, simply supported-simply supported, clamped-guide supported and simply supportedguide supported boundary conditions are examined for the size-dependent free vibration analyses of nanobeams. The finite element method is used to solve the problem. For the vibration response, the effects of the boundary conditions, cross-section (circular and rectangular), material length scale parameter, length-to diameter/height ratio and rotary inertia for different modes on the vibration frequencies are explored. The prominent results obtained may be summarized as follows: For lower material length scale parameter values, the natural frequencies of the rectangular silicon nanobeam are higher than those of the corresponding circular silicon nanobeam. As the lengths of silicon nanobeams increase, the frequency values decrease. Contrary to this situation, the increment of the material length scale parameter results in the increased frequencies. The rotary inertia effect causes a decrease in the frequencies of the nanobeam. In other words, the frequencies obtained via the Rayleigh beam theory are lower than the frequencies obtained via the EulerBernoulli beam theory. Also, the highest frequency values occurred for the $\mathrm{C}-\mathrm{C}$ boundary condition, while the lowest values are seen in the S-G boundary condition.

\section{References}

[1] P. Pasanen, M. Voutilainen, M. Helle, X. Song, P.J. Hakonen, Phys. Scr. 2012, 014025 (2012).

[2] L.A. Dobrzanski, M. Pawlyta, A. Krzton, B. Liszka, C.W. Tai, W. Kwasny, Acta Phys. Pol. A 118, 483 (2010).

[3] I. Pradiptya, H.M. Ouakad, Microsys. Technol. 24, 2585 (2018).

[4] H.Y. Choi, J.U. Kim, C.J. Lee, Acta Phys. Pol. A 115, 1078 (2009).

[5] A.P. Durajski, A.E. Auguścik, Acta Phys. Pol. A 138, 136 (2020). 
[6] T. Mattila, V. Kaajakari, J. Kiihamäki, A. Oja, H. Kattelus, H. Seppä, M. Koskenvuori, P. Rantakari, I. Tittonen, Phys. Scr. 114, 181 (2004).

[7] X. Chen, X. Wang, G.G. Sheng, Phys. Scr. 75, 455 (2007).

[8] R. Ansari, M. Faghih Shojaei, V. Mohammadi, R. Gholami, H. Rouhi, Z. Angew. Math. Mech. 95, 939 (2015).

[9] R. Ansari, J. Torabi, A. Norouzzadeh, Europ. Phys. J. Plus 135, 206 (2020).

[10] Ö. Civalek, B. Uzun, M.Ö. Yayli, B. Akgöz, Europ. Phys. J. Plus 135, 381 (2020).

[11] A. Dihaj, M. Zidour, M. Meradjah, K. Rakrak, H. Heireche, A. Chemi, Struct. Eng. Mech. 65, 335 (2018).

[12] G. Güçlü, R. Artan, Z. Angew. Math. Mech. 100, e201900108 (2020).

[13] Y. Heydarpour, P. Malekzadeh, J. Braz. Soc. Mech. Sci. Eng. 41, 184 (2019).

[14] S.A. Hosseini, F. Khosravi, M. Ghadiri, J. Vibrat. Control 26, 1077546319890170 (2020).

[15] S.M. Hozhabrossadati, N. Challamel, M. Rezaiee-Pajand, A.A. Sani, Mech. Based Des. Struct. Machin., 1 (2020).

[16] C. Li, Mech. Based Des. Struct. Machin. 45, 463 (2017).

[17] B. Uzun, Ö. Civalek, Adv. Nano Res. 7, 99 (2019).

[18] B. Uzun, M.Ö. Yayl,, Int. J. Eng. Appl. Sci. 11, 387 (2019).

[19] B. Uzun, Ö. Civalek, M.Ö. Yaylı, Mech. Based Des. Struct. Machin., 1 (2020).

[20] B. Uzun, M.Ö. Yaylı, Arab. J. Geosci. 13, 1 (2020).

[21] B. Uzun, U. Kafkas, M.Ö. Yaylı, Z. Angew. Math. Mech. 100, e202000039 (2020).

[22] B. Uzun, M.Ö. Yaylı, B. Deliktaş, Micro Nano Lett. 15, 35 (2020).

[23] M.Ö. Yaylı, J. Vibrat. Control 22, 2542 (2016).

[24] M.Ö. Yaylı, J. Brazil. Soc. Mech. Sci. Eng. 40, 419 (2018).

[25] M.Ö. Yayl, Microsyst. Technol. 25, 3723 (2019).

[26] E. Zarezadeh, V. Hosseini, A. Hadi, Mech. Based Des. Struct. Machin. 48, 480 (2019).

[27] N. Challamel, J. Lerbet, C.M. Wang, Z. Zhang, Z. Angew. Math. Mech. 94, 402 (2014).

[28] M.H. Jalaei, Ö. Civalek, Int. J. Eng. Sci. 143, 14 (2019).
[29] Ö. Civalek, B. Uzun, M.Ö. Yaylı, Arab. J. Geosci. 13, 1 (2020).

[30] Ç. Demir, Ö. Civalek, Int. J. Eng. Sci. 121, 14 (2017).

[31] F. Ebrahimi, M.R. Barati, Waves Random Complex Media 28, 326 (2018).

[32] C. Li, H. Guo, X. Tian, Waves Random Complex Media 29, 477 (2019).

[33] M.Ö. Yayli, Acta Phys. Pol. A 127, 678 (2015).

[34] H. Nahvi, M.E. Boroojeni, Acta Phys. Pol. A 123, 304 (2013).

[35] K. Kiani, Acta Phys. Pol. A 131, 1439 (2017).

[36] O. Rahmani, Acta Phys. Pol. A 125, 532 (2014).

[37] S. Kong, S. Zhou, Z. Nie, K. Wang, Int. J. Eng. Sci. 47, 487 (2009).

[38] J. Lei, Y. He, B. Zhang, Z. Gan, P. Zeng, Int. J. Eng. Sci. 72, 36 (2013).

[39] K.A. Lazopoulos, A.K. Lazopoulos, Z. Angew. Math. Mech. 91, 875 (2011).

[40] B. Wang, M. Liu, J. Zhao, S. Zhou, Meccanica 49, 1427 (2014).

[41] L. Zhang, B. Liang, S. Zhou, B. Wang, Y. Xue, Meccanica 52, 251 (2017).

[42] M.Ö. Yaylı, B. Uzun, B. Deliktaş, Waves Random Complex Media, 1 (2021).

[43] B. Akgöz, Ö. Civalek, Int. J. Mech. Sci. 99, 10 (2015).

[44] F. Ebrahimi, M.R. Barati, Ö. Civalek, Eng. Comput. 36, 953 (2020).

[45] S.K. Jena, S. Chakraverty, M. Malikan, F. Tornabene, Mech. Based Des. Struct. Machin. 49, 581 (2021).

[46] M.A. Eltaher, F.A. Omar, W.S. Abdalla, E.H. Gad, Waves Random Complex Media 29, 264 (2019).

[47] R. Bahaadini, A.R. Saidi, M. Hosseini, J. Vibrat. Control 25, 203 (2019).

[48] S.K. Jena, S. Chakraverty, M. Malikan, Eng. Comput. 37, 1 (2019).

[49] B. Karami, D. Shahsavari, M. Janghorban, R. Dimitri, F. Tornabene, Nanomaterials 9, 22 (2019).

[50] H.B. Khaniki, S. Hosseini-Hashemi, Mater. Res. Express 4, 065003 (2017).

[51] Q. Wu, H. Chen, W. Gao, Eng. Comput. 36, 1739 (2020).

[52] A. Gholipour, M.H. Ghayesh, Microsyst. Technol. 26, 2053 (2020).

[53] S. Sahmani, A. Khandan, Microsyst. Technol. 25, 2171 (2019).

[54] A. Babaei, Microsyst. Technol. 27, 821 (2021). 
[55] K. Mohammadi, M. Mahinzare, K. Ghorbani, M. Ghadiri, Microsyst. Technol. 24, 1133 (2018).

[56] F. Ebrahimi, M.R. Barati, Microsyst. Technol. 24, 1643 (2018).

[57] B. Alizadeh Hamidi, F. Khosravi, S.A. Hosseini, R. Hassannejad, Waves Random Complex Media, 1 (2020).

[58] M. Mahinzare, K. Mohammadi, M. Ghadiri, Waves Random Complex Media 31, 305 (2021).

[59] F.A.C.M. Yang, A.C.M. Chong, D.C.C. Lam, P. Tong, Int. J. Solids Struct. 39, 2731 (2002).

[60] A. Ghanbari, A. Babaei, Int. Res. J. Appl. Basic Sci. 9, 274 (2015).

[61] N. Togun, S.M. Bağdatli, Tehnički glasnik 12, 221 (2018).

[62] S.K. Park, X.L. Gao, J. Micromech. Microeng. 16, 2355 (2006).

[63] S. Kong, S. Zhou, Z. Nie, K. Wang, Int. J. Eng. Sci. 46, 427 (2008).

[64] Ö. Civalek, S. Dastjerdi, Ş.D. Akbaş, B. Akgöz, Math. Meth. Appl. Sci., 1 (2021).

[65] H. Ersoy, H.M. Numanoğlu, B. Akgöz, Ö. Civalek, Curv. Layer. Struct. 5, 273 (2018).

[66] A.R. Daneshmehr, M. Mohammad Abadi, A. Rajabpoor, Appl. Mech. Mater. 332 , 331 (2013).
[67] L.L. Ke, Y.S. Wang, Z.D. Wang, Physica E 43, 1387 (2011).

[68] R. Ansari, M.A. Ashrafi, S. Hosseinzadeh, Shock Vibrat. 2014, 598292 (2014).

[69] O. Mazur, L. Kurpa, J. Awrejcewicz, Z. Angew. Math. Mech. 100, e202000009 (2020).

[70] Ş.D. Akbaş, Smart Struct. Syst. 18, 1125 (2016).

[71] B. Uzun, U. Kafkas, M.Ö. Yayl, Microsyst. Technol. 27, 1913 (2021).

[72] M. Asghari, M.H. Kahrobaiyan, M.T. Ahmadian, Int. J. Eng. Sci. 48, 1749 (2010).

[73] M.H. Ghayesh, H. Farokhi, M. Amabili, Composites B 50, 318 (2013).

[74] Y.G. Wang, W.H. Lin, N. Liu, Physica E 47, 80 (2013).

[75] N. Togun, S.M. Bağdatli, Composites B 97, 255 (2016).

[76] J.N. Reddy, Energy Principles and Variational Methods in Applied Mech., 2nd ed., Wiley, New York 2002.

[77] J.N. Reddy, An Introduction to the Finite Element Method, 2nd ed., McGraw-Hill, New York 1993.

[78] R. Zhu, E. Pan, P.W. Chung, X. Cai, K.M. Liew, A. Buldum, Semicond. Sci. Technol. 21, 906 (2006).

[79] S. Hosseini-Hashemi, M. Fakher, R. Nazemnezhad, J. Solid Mech. 5, 290 (2013). 